\title{
Response of renal cell carcinoma to ibrutinib, a bruton tyrosine kinase inhibitor, in a patient treated for chronic lymphocytic leukemia
}

\author{
Gregory W. Hosier, MD; Naji J. Touma, MD
}

Department of Urology, Queen's University, Kingston, ON, Canada

Cite as: Can Urol Assoc J 2017; 11 (5):E237-9. http://dx.doi.org/10.5489/cuaj.4316

Published online May 9, 2017

\section{Introduction}

Ibrutinib is a bruton tyrosine kinase (BTK) inhibitor approved for B cell malignancies. Although there are currently two clinical trials evaluating ibrutinib in combination with nivolumab (programmed cell death protein 1, PD-1, inhibitor) or everolimus (mammalian target of rapamycin, mTOR, inhibitor) for metastatic renal cell carcinoma (RCC), there are no reports of RCC (metastatic or non-metastatic) showing response to a BTK inhibitor in humans. Here we report a 22-month clinical response of biopsy-proven RCC to ibrutinib. This is unexpected, given that BTK is not wellimplicated in RCC pathophysiology. We explore a possible mechanism for the response of RCC to ibrutinib through inhibition of interleukin-2-inducible T-cell kinase (ITK) leading to enhanced antitumour immune responses.

\section{Case report}

A 66-year-old male was referred for a left renal mass. His past medical history was significant for newly diagnosed chronic lymphocytic leukemia (CLL) with 17 p chromosomal deletions. At the time of CLL diagnosis, he had palpable lymphadenopathy in the left posterior cervical triangle, both axillae, and significant pelvic lymphadenopathy causing bladder obstruction requiring catheterization. He denied hematuria, flank pain, flank mass, or weight loss. He had a significant smoking history. Blood work revealed hemoglobin $136 \mathrm{~g} / \mathrm{L}$, white blood cell count $20.9 \times 10^{9} / \mathrm{L}$, platelets 138 x $10^{9} / \mathrm{L}$, and creatinine $98 \mu \mathrm{mol} / \mathrm{L}$. Computed tomography (CT) scan showed a left renal $5.3 \times 4.7 \times 4.5 \mathrm{~cm}$ mesophytic interpolar well-circumscribed mass. The appearance was felt to be most consistent with RCC, but lymphoma could not be ruled out. A biopsy was performed and showed RCC of unknown subtype. Given his significant symptom burden and uncertain prognosis, he was started on therapy for CLL and further management of his RCC was postponed.

Under the care of medical oncology, he underwent six cycles of fludarabine, cyclophosphamide, and rituximab. Repeat CT scan six months later showed increase in size of the renal mass to $6.3 \times 5.7 \times 5.1 \mathrm{~cm}$ (Fig. 1). Due to incomplete response of his CLL to chemotherapy, he was started on ibrutinib $420 \mathrm{mg}$ orally daily. Repeat CT scan one month later showed decrease of the renal mass to $4.8 \times 4.6 \times 4.5$ $\mathrm{cm}$. Subsequent CT scans continued to show a progressive decrease of the size of the renal mass. The lymphadenopathy related to the CLL improved to the point where all previously enlarged lymph nodes were below $1 \mathrm{~cm}$. His last CT scan 22 months after the start of ibrutinib showed the left renal mass had shrunk to $2.3 \times 1.8 \times 2.4 \mathrm{~cm}$ (Fig. 1). He continues on ibrutinib for his CLL and his continuously shrinking renal mass is under an active surveillance protocol.

\section{Discussion}

Here we report a roughly 22-month clinical response of non-metastatic RCC to ibrutinib, a BTK inhibitor. Currently, there are two clinical trials involving ibrutinib and metastatic RCC. NCT02899078 is a phase $1 / 2$ b trial evaluating safety and efficacy of a combination of ibrutinib and nivolumab (programmed cell death protein 1, PD-1, inhibitor) in patients with metastatic RCC that previously completed at least one systemic therapy. NCT02599324 is a phase 1/2 trial evaluating safety and efficacy of ibrutinib with everolimus (mammalian target of rapamycin, mTOR, inhibitor) in patients with metastatic RCC previously treated with at least one systemic therapy. To the best of our knowledge, this is the first report of RCC (non-metastatic or metastatic) showing a response to a BTK inhibitor.

BTK is a cytoplasmic, non-receptor tyrosine kinase that is an essential component of B cell receptor signaling. ${ }^{1}$ Activation of BTK through phosphorylation of its active site 

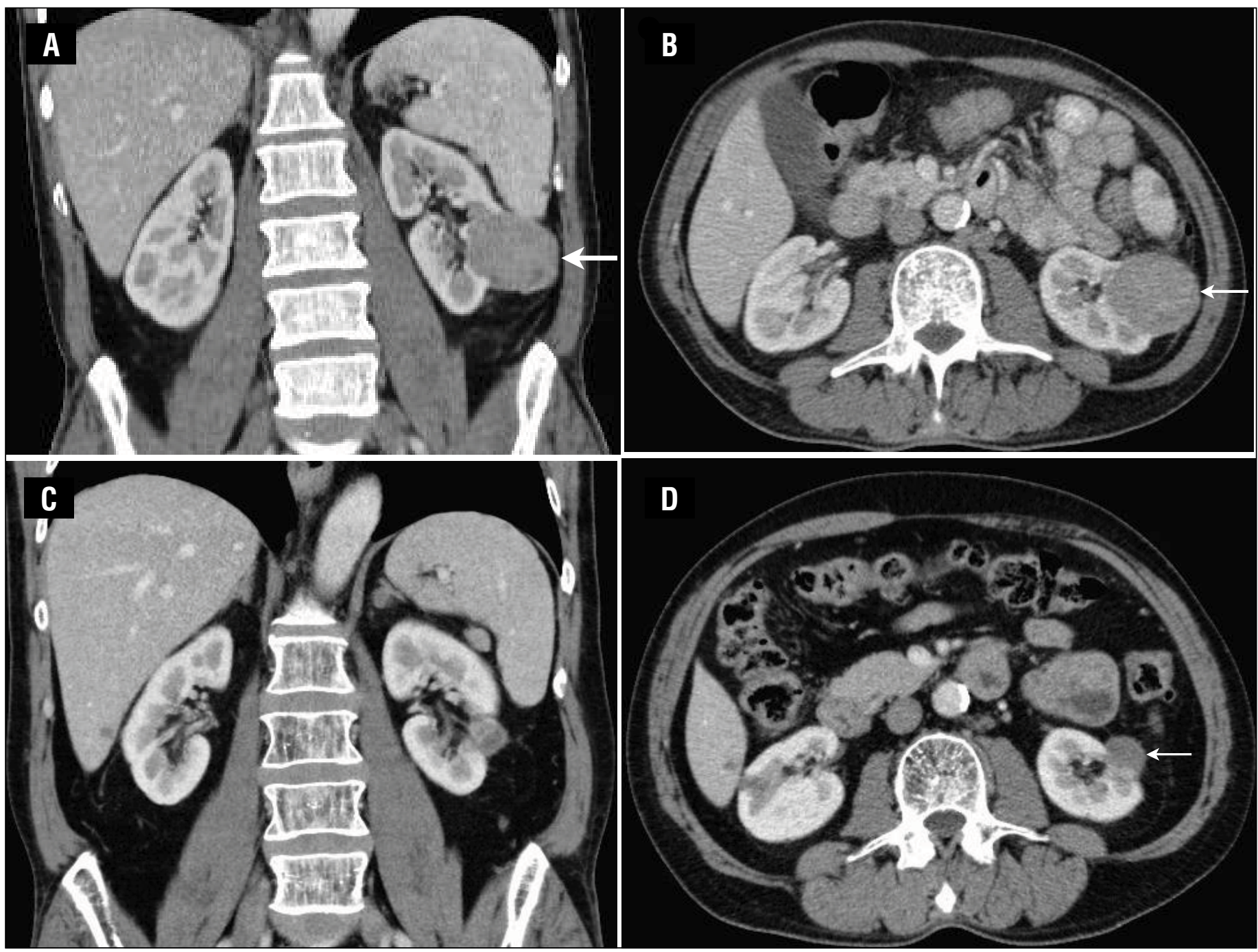

Fig.1. Enhanced abdominal computed tomography scan showing biopsy proven renal cell carcinoma (arrow) prior to treatment with ibrutinib $(\boldsymbol{A}, \boldsymbol{B}: 6.3 \times 5.7 \times 5.1 \mathrm{~cm})$ and 22 months post-initiation of ibrutinib (C, D: $2.3 \times 1.8 \times 2.4 \mathrm{~cm}$ ).

at cysteine 481 leads to subsequent activation of the phosphoinositol-3 kinase/AKT, phospholipase-C, protein kinase $\mathrm{C}$, and nuclear factor kappa B pathways that ultimately leads to B cell differentiation, proliferation, and survival. ${ }^{1}$ Ibrutinib inhibits BTK by covalently binding to the active site and abolishing B cell receptor signaling downstream of BTK. ${ }^{2}$ Only a small subset of tyrosine kinases in the human genome is predicted to contain a modifiable cysteine residue homologous to cysteine 481 in BTK..$^{1,3}$ These include EGFR, HER2, HER4, ITK, BMX, JAK3, TEC, and BLK. ${ }^{1,3}$ To what extent inhibition of these kinases contributes to efficacy of ibrutinib is unknown. Ibrutinib was approved by the Federal Drug Agency for treatment of B cell malignancies including CLL, mantle cell lymphoma, multiple myeloma, diffuse large B cell lymphoma, and Waldenström's macroglobulinemia between 2013 and 2015. ${ }^{4}$ Ibrutinib is approved in Canada as first-line treatment for CLL patients with $17 p$ deletion, who historically have responded poorly to chemotherapy. ${ }^{5}$

Although BTK is not well-implicated in RCC pathophysiology, there is evidence from cell and animal models that ibrutinib inhibits interleukin-2-ITK, which could modulate RCC progression. ITK is a cellular tyrosine kinase that is highly expressed in T cells. ${ }^{6}$ ITK inhibition by ibrutinib is believed to shift the balance between Th1 and Th2 T cells and enhance antitumour immune responses. ${ }^{7-9}$ This mechanism is believed to potentiate immunomodulatory effects of checkpoint inhibitors nivolumab and ipilimumab (inhibitor of cytotoxic T-lymphocyte-associated protein, CTLA4). ${ }^{3,7}$ Checkpoint inhibitors are an emerging therapeutic modality for metastatic RCC..$^{10,11}$ In a phase 3 clinical trial 
(NCT01668784) for patients with metastatic RCC who failed previous treatment, patients treated with nivolumab had increased overall survival compared to everolimus (25.0 months vs. 19.6 months, respectively). ${ }^{12}$ It is possible that the beneficial effects of ibrutinib on RCC described here were due in part to inhibition of ITK leading to enhanced antitumour immune responses, possibly through inhibition of PD-1 or CTLA-4.

\section{Conclusion}

We report a 22-month clinical response of non-metastatic RCC to ibrutinib. Although there are currently two clinical trials evaluating ibrutinib in combination with nivolumab or everolimus for metastatic RCC, this is the first report of RCC (metastatic or non-metastatic) showing response to a BTK inhibitor in humans. Further studies investigating the impact of ibrutinib on progression of non-metastatic and metastatic RCC alone or in combination with other treatments may be warranted.

Competing interests: The authors report no competing personal or financial interests.

This paper has been peer-reviewed.

\section{References}

1. Ponader S, Burger JA. Bruton's tyrosine kinase: From X-linked agammaglobulinemia toward targeted therapy for B-cell malignancies. J Clin Oncol 2014;17:1830-9. https://doi.org/10.1200/JC0.2013.53.1046

2. Lee C-S, Rattu MA, Kim SS. A review of a novel, Brutons tyrosine kinase inhibitor, ibrutinib. J Oncol Pharm Pract 2016;22:92-104. https://doi.org/10.1177/1078155214561281

3. Berglöf A, Hamasy A, Meinke S, et al. Targets for ibrutinib beyond B cell malignancies. Scand I Immunol 2015;82:208-17. https://doi.org/10.1111/sii.12333

4. Kim ES, Dhillon S. Ibrutinib: A review of its use in patients with mantle cell lymphoma or chronic lymphocytic leukemia. Drugs 2015;75:769-76. https://doi.org/10.1007/s40265-015-0380-3

5. Owen C, Bence-Brucker I, Chamakhi I, et al. A Canadian perspective on the first-line treatment of chronic lymphocytic leukemia. Clin Lymphoma, Myeloma Leuk 2015;15:303-13. https://doi.org/10.1016/i. clml.2015.03.002

6. Andreotti AH, Schwartzberg PL, Joseph RE, et al. T-cell signaling regulated by the Tec family kinase, Itk. Cold Spring Harb Perspect Biol 2010;2:0002287-0002287. https://doi.org/10.1101/cshperspect. 0002287

7. Sagiv-Barfi I, Kohrt HEK, Czerwinski DK, et al. Therapeutic antitumor immunity by checkpoint blockade is enhanced by ibrutinib, an inhibitor of both BTK and ITK. Proc Natl Acad Sci USA 2015;112:E966-72. https://doi.org/10.1073/pnas.1500712112

8. Dubovsky JA, Beckwith KA, Natarajan $G$, et al. Ibrutinib is an irreversible molecular inhibitor of ITK driving a Th1-selective pressure in Tlymphocytes. Blood 2013;122:2539-49. https://doi.org/10.1182/ blood-2013-06-507947

9. Schutt SD, Fu J, Nguyen $\mathrm{H}$, et al. Inhibition of BTK and ITK with ibrutinib is effective in the prevention of chronic graft-vs-host disease in mice. PLoS One 2015;10:e0137641. https://doi.org/10.1371/ journal.pone.0137641

10. Carlo MI, Voss MH, Motzer RJ. Checkpoint inhibitors and other novel immunotherapies for advanced renal cell carcinoma. Nat Rev Urol 2016;13:420-31. https://doi.org/10.1038/nrurol.2016.103

11. Lee C-H, Motzer RJ. Immune checkpoint therapy in renal cell carcinoma. Cancer J 2016;22:92-5. https://doi.org/10.1097/PP0.0000000000000177

12. Motzer RJ, Escudier B, McDermott DF, et al. Nivolumab vs. everolimus in advanced renal cell carcinoma. N Engl J Med 2015;373:1803-13. https://doi.org/10.1056/NEJMoa1510665

Correspondence: Dr. Gregory W. Hosier, Department of Urology, Queen's University, Kingston, ON, Canada; gregory.hosier@gmail.com 